OPEN Ә ACCESS

드 COMMUNICATIONS

ISSN 2056-9890

\section{Crystal structure of 3-(9H-carbazol-9-yl)- $N^{\prime}$-[(E)-4-chlorobenzylidene]propano- hydrazide}

Mehmet Akkurt, ${ }^{a}$ Jerry P. Jasinski, ${ }^{\text {b }}$ Shaaban K. Mohamed, ${ }^{\mathrm{c}, \mathrm{d}}$ Talaat I. El-Emary ${ }^{\mathrm{e}}$ and Mustafa R. Albayati ${ }^{\mathrm{f}}$ *

aDepartment of Physics, Faculty of Sciences, Erciyes University, 38039 Kayseri, Turkey, ${ }^{\mathbf{b}}$ Department of Chemistry, Keene State College, 229 Main Street, Keene, NH 03435-2001, USA, 'cChemistry and Environmental Division, Manchester Metropolitan University, Manchester M1 5GD, England, 'Chemistry Department, Faculty of Science, Minia University, 61519 El-Minia, Egypt, ${ }^{\mathbf{e}}$ Department of Chemistry, Faculty of Science, Assiut University, 71515 Assiut, Egypt, and 'Kirkuk University, College of Science, Department of Chemistry, Kirkuk, Iraq. *Correspondence e-mail: shaabankamel@yahoo.com

Received 30 October 2015; accepted 2 November 2015

Edited by P. McArdle, National University of Ireland, Ireland

In the title compound, $\mathrm{C}_{22} \mathrm{H}_{18} \mathrm{ClN}_{3} \mathrm{O}$, the carbazole ring system is essentially planar (r.m.s deviation $=0.003 \AA$ ), and makes a dihedral angle of $9.01(8)^{\circ}$ with the plane of the chlorophenyl ring. In the crystal, neighbouring molecules are linked into centrosymmetric $R_{2}^{2}(8)$ dimers by pairs of $\mathrm{N}-$ $\mathrm{H} \cdots \mathrm{O}$ interactions and into a three-dimensional network by $\mathrm{C}-\mathrm{H} \cdots \pi$ interactions. The dimers are arranged into layers parallel to (010).

Keywords: crystal structure; the carbazole ring system; bio-active molecules; hydrogen bonding.

CCDC reference: 1434700

\section{Related literature}

For synthesis and pharmacuetical studies of carbazole containing compounds, see: Hewlins et al. (1984); Kansal \& Potier (1986); Haider et al. (1998); Hirata et al. (1999); Chowdhury et al. (1978); Sakano et al. (1980); Pindur (1990); Knölker \& Reddy (2002); Martin \& Prasad (2006); Saturnino et al. (2003).

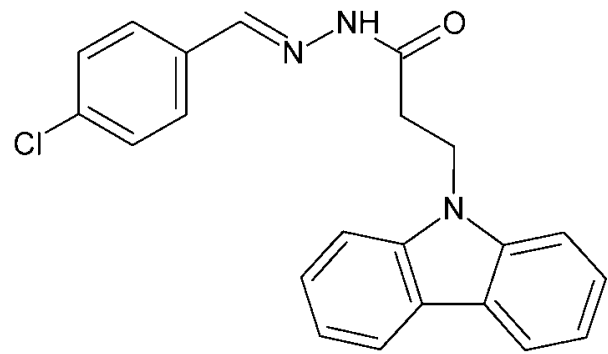

2. Experimental

2.1. Crystal data

$\mathrm{C}_{22} \mathrm{H}_{18} \mathrm{ClN}_{3} \mathrm{O}$

$M_{r}=375.84$

Monoclinic, $P 2_{1} / c$

$a=16.0126(7) \AA$

$b=7.4316(3) \AA$

$c=16.1654(9) \AA$

$\beta=94.607$ (4)

$$
\begin{aligned}
& V=1917.46(16) \AA^{3} \\
& Z=4 \\
& \text { Mo } K \alpha \text { radiation } \\
& \mu=0.22 \mathrm{~mm}^{-1} \\
& T=293 \mathrm{~K} \\
& 0.42 \times 0.36 \times 0.08 \mathrm{~mm}
\end{aligned}
$$

\subsection{Data collection}

Agilent Xcalibur Eos Gemini diffractometer

Absorption correction: multi-scan (CrysAlis PRO; Agilent, 2014)

$$
T_{\min }=0.847, T_{\max }=1.000
$$

12277 measured reflections 6312 independent reflections 3066 reflections with $I>2 \sigma(I)$ $R_{\text {int }}=0.023$

\subsection{Refinement}

$R\left[F^{2}>2 \sigma\left(F^{2}\right)\right]=0.058$

$w R\left(F^{2}\right)=0.167$

$S=1.02$

6312 reflections
244 parameters

$\mathrm{H}$-atom parameters constrained $\Delta \rho_{\max }=0.20 \mathrm{e} \AA^{-3}$

$\Delta \rho_{\min }=-0.24$ e $\AA^{-3}$
Table 1

Hydrogen-bond geometry $\left(\AA{ }^{\circ}\right)$.

$\mathrm{Cg} 2, \mathrm{Cg} 3$ and $\mathrm{Cg} 4$ are the centroids of the two benzene rings (C1-C6 and C7$\mathrm{C} 12)$ of the carbazole ring system and the chlorophenyl ring (C17-C22), respectively.

\begin{tabular}{lllll}
\hline$D-\mathrm{H} \cdots A$ & $D-\mathrm{H}$ & $\mathrm{H} \cdots A$ & $D \cdots A$ & $D-\mathrm{H} \cdots A$ \\
\hline $\mathrm{N} 2-\mathrm{H} 2 N \cdots \mathrm{O} 1^{\mathrm{i}}$ & 0.81 & 2.08 & $2.8952(19)$ & 175 \\
$\mathrm{C} 5-\mathrm{H} 5 \cdots C g 4^{\text {ii }}$ & 0.93 & 2.81 & $3.696(3)$ & 160 \\
$\mathrm{C} 21-\mathrm{H} 21 \cdots C g 3^{\text {iii }}$ & 0.93 & 2.97 & $3.858(3)$ & 160 \\
$\mathrm{C} 22-\mathrm{H} 22 \cdots C g 2^{\text {iii }}$ & 0.93 & 2.79 & $3.699(2)$ & 166 \\
\hline
\end{tabular}

Symmetry codes: (i) $-x+1,-y-1,-z+1$; (ii) $x,-y+\frac{1}{2}, z+\frac{1}{2}$; (iii) $x,-y-\frac{1}{2}, z-\frac{1}{2}$.

Data collection: CrysAlis PRO (Agilent, 2014); cell refinement: CrysAlis PRO; data reduction: CrysAlis PRO; program(s) used to solve structure: SIR92 (Altomare et al., 1999); program(s) used to refine structure: SHELXL2014 (Sheldrick, 2015); molecular graphics: ORTEP-3 for Windows (Farrugia, 2012); software used to prepare material for publication: PLATON (Spek, 2009).

\section{Acknowledgements}

JPJ acknowledges the NSF-MRI program (grant No. CHE1039027) for funds to purchase the X-ray diffractometer. 


\section{data reports}

Supporting information for this paper is available from the $\mathrm{IUCr}$ electronic archives (Reference: QM2112).

\section{References}

Agilent (2014). CrysAlis PRO. Agilent Technologies Ltd, Yarnton, England. Altomare, A., Burla, M. C., Camalli, M., Cascarano, G. L., Giacovazzo, C., Guagliardi, A., Moliterni, A. G. G., Polidori, G. \& Spagna, R. (1999). J. Appl. Cryst. 32, 115-119.

Chowdhury, D. N., Basak, S. K. \& Das, B. P. (1978). Curr. Sci. 47, 490-491

Farrugia, L. J. (2012). J. Appl. Cryst. 45, 849-854.

Haider, N., Jbara, R., Khadami, F. \& Wanko, R. (1998). Heterocycles, 48, 16091622
Hewlins, M. J. E., Oliveira-Campos, A. \& Shannon, P. V. R. (1984). Synthesis, pp. 289-302.

Hirata, K., Ito, C., Furukawa, H., Itoigawa, M., Cosentino, L. M. \& Lee, K. H. (1999). Bioorg. Med. Chem. Lett. 9, 119-122.

Kansal, V. K. \& Potier, P. (1986). Tetrahedron, 42, 2389-2408.

Knölker, H. J. \& Reddy, K. R. (2002). Chem. Rev. 102, 4303-4428.

Martin, A. E. \& Prasad, K. J. R. (2006). Acta Pharm. 56, 79-86.

Pindur, U. (1990). Chimia, 44, 406-412.

Sakano, K., Ishimaru, K. \& Nakamura, S. (1980). J. Antibiot. 33, 683-689.

Saturnino, C., Buonerba, M., Boatto, G., Pascale, M., Moltedo, O., de Napoli, L., Montesarchio, D., Lancelot, J. C. \& de Caprariis, P. (2003). Chem. Pharm. Bull. 51, 971-974.

Sheldrick, G. M. (2015). Acta Cryst. C71, 3-8.

Spek, A. L. (2009). Acta Cryst. D65, 148-155. 


\section{supporting information}

Acta Cryst. (2015). E71, o937-o938 [https://doi.org/10.1107/S2056989015020770]

\section{Crystal structure of 3-(9H-carbazol-9-yl)-N'-[(E)-4-chlorobenzylidene]propano- hydrazide}

Mehmet Akkurt, Jerry P. Jasinski, Shaaban K. Mohamed, Talaat I. El-Emary and Mustafa R. Albayati

\section{S1. Comment}

Carbazole scaffold compunds are well known for their pharmacological activities. The syntheses of carbazole derivatives in connection with the search for newer physiologically activities have been recognized in many reports (Hewlins et al., 1984; Kansal \& Potier 1986; Haider et al., 1998; Hirata et al., 1999). Carbazomycin A and carbazomycin B have been found to be useful antibacterial and antifungal agents (Chowdhury et al., 1978; Sakano et al., 1980). In addition pyridocarbazoles show marked anticancer and anti-HIV activities (Pindur, 1990; Knölker \& Reddy, 2002; Martin \& Prasad 2006; Saturnino et al., 2003). Based on such facts we report in this study the synthesis and crystal structure of the title compound.

As shown in Fig. 1, the carbazole ring system (N1/C1-C12) of the title compound is essentially planar (r.m.s deviation $=0.003 \AA$ ), and makes a dihedral angle of $9.01(8)^{\circ}$ with the plane of the chlorophenyl ring $(\mathrm{C} 17-\mathrm{C} 22)$. The bond lengths and angles are within normal ranges and are similar to those reported earlier for similar compounds.

In the crystal, two molecules are associated through a pair of $\mathrm{N}-\mathrm{H} \cdots \mathrm{O}$ intermolecular hydrogen bonds, forming a centrosymmetric dimer with $R_{2}{ }^{2}(8)$ ring motifs (Table 1), into layers parallel to (010) (Fig. 2). The dimers are connected by $\mathrm{C}-\mathrm{H} \cdots \pi$ interactions, forming a three-dimensional network.

\section{S2. Experimental}

A mixture of $1.5 \mathrm{mmol}(380 \mathrm{mg})$ of 3-(9H-carbazol-9-yl)propanehydrazide and $1.5 \mathrm{mmol}(261 \mathrm{mg})$ of 4-chlorobenzaldehyde was heated in $10 \mathrm{ml}$ of absolute ethanol and $3 \mathrm{ml}$ of acetic acid catalyst. The reaction was monitored by TLC till completion after $3 \mathrm{~h}$. The product which deposited on cooling, was collected, dried under vacuum and recrystallized from dioxan to give orange plates in $78 \%$ yield.

\section{S3. Refinement}

All $\mathrm{H}$ atoms were placed in calculated positions with $\mathrm{N}-\mathrm{H}=0.81$ and $\mathrm{C}-\mathrm{H}=0.93-0.97 \AA$, and refined as riding with $U_{\mathrm{iso}}(\mathrm{H})=1.2 U_{\mathrm{eq}}(\mathrm{C}, \mathrm{N})$. 


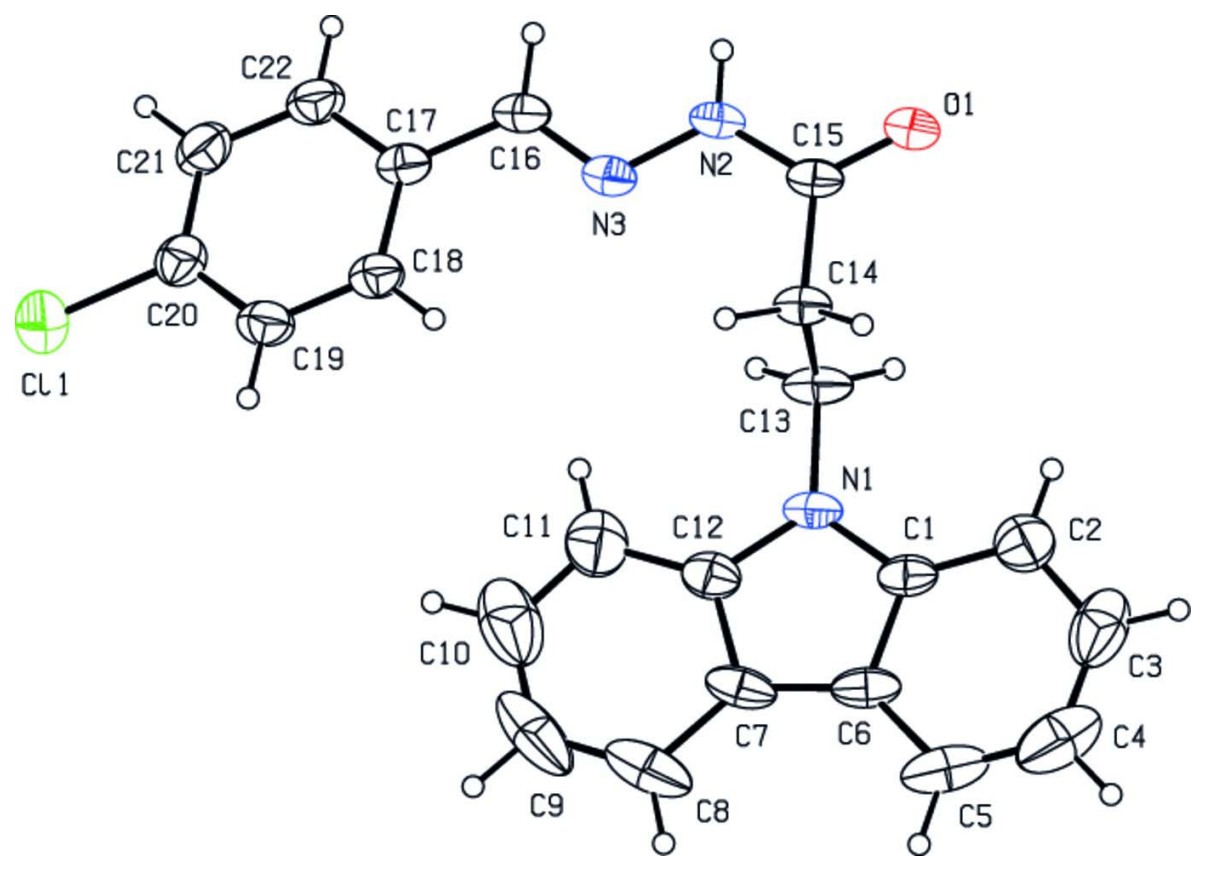

Figure 1

View of the title compound with the atom numbering scheme. Displacement ellipsoids for non- $\mathrm{H}$ atoms are drawn at the $30 \%$ probability level.

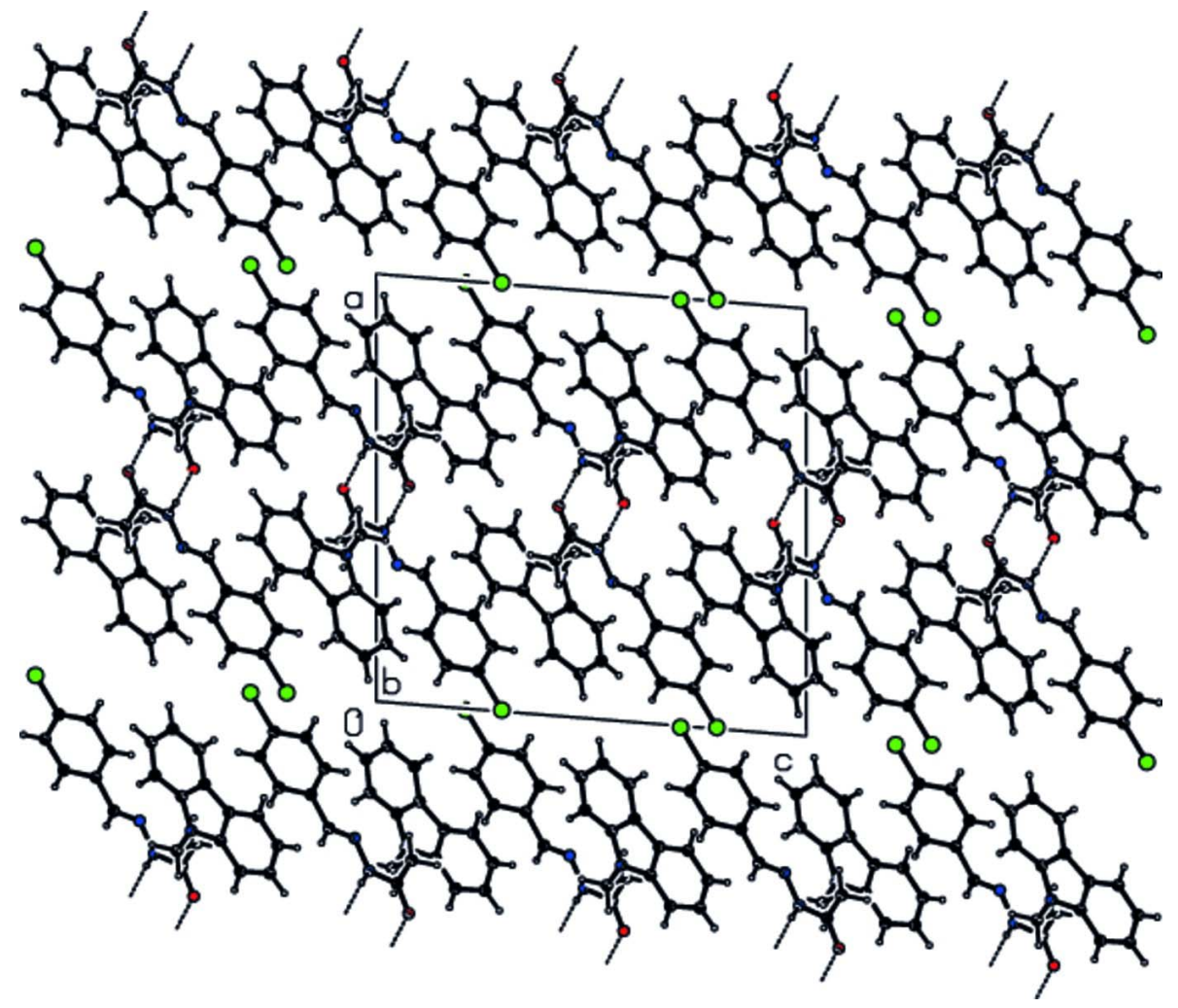

Figure 2

View of the dimers formed by $\mathrm{N}-\mathrm{H} \cdots \mathrm{O}$ hydrogen bonds down the $b$ axis. 
3-(9H-Carbazol-9-yl)-N'-[(E)-4-chlorobenzylidene]propanohydrazide

Crystal data

$\mathrm{C}_{22} \mathrm{H}_{18} \mathrm{ClN}_{3} \mathrm{O}$

$M_{r}=375.84$

Monoclinic, $P 2{ }_{1} / c$

Hall symbol: -P $2 \mathrm{ybc}$

$a=16.0126(7) \AA$

$b=7.4316(3) \AA$

$c=16.1654(9) \AA$

$\beta=94.607(4)^{\circ}$

$V=1917.46(16) \AA^{3}$

$Z=4$

\section{Data collection}

Agilent Xcalibur Eos Gemini diffractometer

Radiation source: Enhance (Mo) X-ray Source

Graphite monochromator

Detector resolution: 16.0416 pixels $\mathrm{mm}^{-1}$

$\omega$ scans

Absorption correction: multi-scan

(CrysAlis PRO; Agilent, 2014)

$T_{\min }=0.847, T_{\max }=1.000$

Refinement

Refinement on $F^{2}$

Least-squares matrix: full

$R\left[F^{2}>2 \sigma\left(F^{2}\right)\right]=0.058$

$w R\left(F^{2}\right)=0.167$

$S=1.02$

6312 reflections

244 parameters

0 restraints

Special details

Geometry. Bond distances, angles etc. have been calculated using the rounded fractional coordinates. All su's are estimated from the variances of the (full) variance-covariance matrix. The cell e.s.d.'s are taken into account in the estimation of distances, angles and torsion angles

Refinement. Refinement on $F^{2}$ for ALL reflections except those flagged by the user for potential systematic errors. Weighted $R$-factors $w R$ and all goodnesses of fit $S$ are based on $F^{2}$, conventional $R$-factors $R$ are based on $F$, with $F$ set to zero for negative $F^{2}$. The observed criterion of $F^{2}>\sigma\left(F^{2}\right)$ is used only for calculating -R-factor-obs etc. and is not relevant to the choice of reflections for refinement. $R$-factors based on $F^{2}$ are statistically about twice as large as those based on $F$, and $R$-factors based on ALL data will be even larger.

Fractional atomic coordinates and isotropic or equivalent isotropic displacement parameters $\left(\hat{A}^{2}\right)$

\begin{tabular}{lllll}
\hline & $x$ & $y$ & $z$ & $U_{\text {iso }} * / U_{\text {eq }}$ \\
\hline C11 & $0.99666(4)$ & $-0.31996(9)$ & $0.20792(5)$ & $0.0942(3)$ \\
O1 & $0.50960(9)$ & $-0.32276(16)$ & $0.57559(9)$ & $0.0624(5)$ \\
N1 & $0.66300(10)$ & $0.17932(18)$ & $0.56956(10)$ & $0.0547(5)$ \\
N2 & $0.60196(9)$ & $-0.39049(19)$ & $0.48394(9)$ & $0.0535(5)$ \\
N3 & $0.67596(9)$ & $-0.35405(19)$ & $0.44990(10)$ & $0.0511(5)$
\end{tabular}




\begin{tabular}{|c|c|c|c|c|}
\hline $\mathrm{C} 1$ & $0.64514(12)$ & $0.2806(2)$ & $0.63777(12)$ & $0.0539(6)$ \\
\hline $\mathrm{C} 2$ & $0.57672(16)$ & $0.2737(3)$ & $0.68399(17)$ & $0.0803(9)$ \\
\hline $\mathrm{C} 3$ & $0.5780(2)$ & $0.3896(4)$ & $0.75265(19)$ & $0.1108(14)$ \\
\hline $\mathrm{C} 4$ & $0.6440(3)$ & $0.5071(4)$ & $0.77117(19)$ & $0.1203(16)$ \\
\hline $\mathrm{C} 5$ & $0.7099(2)$ & $0.5122(3)$ & $0.72554(17)$ & $0.0944(12)$ \\
\hline C6 & $0.71287(14)$ & $0.3976(2)$ & $0.65793(12)$ & $0.0609(7)$ \\
\hline $\mathrm{C} 7$ & 0.77393 & $0.3604(3)$ & $0.60061(13)$ & $0.0649(7)$ \\
\hline $\mathrm{C} 8$ & $0.85479(19)$ & $0.4244(4)$ & $0.5889(2)$ & $0.1049(13)$ \\
\hline C9 & $0.8972(2)$ & $0.3490(6)$ & $0.5267(3)$ & $0.1362(18)$ \\
\hline $\mathrm{C} 10$ & $0.8626(3)$ & $0.2141(6)$ & $0.4766(3)$ & $0.1306(17)$ \\
\hline $\mathrm{C} 11$ & $0.78534(18)$ & 0.1509 (4) & $0.48540(17)$ & $0.0887(10)$ \\
\hline $\mathrm{C} 12$ & $0.74149(13)$ & $0.2233(2)$ & $0.54750(13)$ & $0.0578(6)$ \\
\hline $\mathrm{C} 13$ & $0.61339(13)$ & $0.0290(2)$ & $0.53681(13)$ & $0.0646(7)$ \\
\hline $\mathrm{C} 14$ & $0.63465(11)$ & $-0.1461(2)$ & $0.58248(12)$ & $0.0522(6)$ \\
\hline $\mathrm{C} 15$ & $0.57783(12)$ & $-0.2941(2)$ & $0.54829(12)$ & $0.0502(6)$ \\
\hline $\mathrm{C} 16$ & $0.69414(11)$ & $-0.4544(2)$ & $0.39006(12)$ & $0.0546(6)$ \\
\hline $\mathrm{C} 17$ & $0.76989(11)$ & $-0.4242(2)$ & $0.34818(11)$ & $0.0507(6)$ \\
\hline $\mathrm{C} 18$ & $0.83316(12)$ & $-0.3082(2)$ & $0.37949(12)$ & $0.0567(6)$ \\
\hline $\mathrm{C} 19$ & $0.90254(12)$ & $-0.2777(3)$ & $0.33665(14)$ & $0.0625(7)$ \\
\hline $\mathrm{C} 20$ & $0.90972(13)$ & $-0.3622(3)$ & $0.26225(14)$ & $0.0626(7)$ \\
\hline $\mathrm{C} 21$ & $0.84950(14)$ & $-0.4801(3)$ & $0.23015(14)$ & $0.0701(8)$ \\
\hline $\mathrm{C} 22$ & $0.78026(13)$ & $-0.5105(3)$ & $0.27385(13)$ & $0.0647(7)$ \\
\hline $\mathrm{H} 2$ & 0.53200 & 0.19640 & 0.67050 & $0.0960 *$ \\
\hline $\mathrm{H} 2 \mathrm{~N}$ & 0.57290 & -0.47400 & 0.46610 & $0.0640 *$ \\
\hline H3 & 0.53370 & 0.38750 & 0.78640 & $0.1330^{*}$ \\
\hline $\mathrm{H} 4$ & 0.64240 & 0.58410 & 0.81640 & $0.1450^{*}$ \\
\hline H5 & 0.75350 & 0.59220 & 0.73900 & $0.1130 *$ \\
\hline $\mathrm{H} 8$ & 0.87900 & 0.51530 & 0.62240 & $0.1260 *$ \\
\hline H9 & 0.95070 & 0.39030 & 0.51840 & $0.1640 *$ \\
\hline $\mathrm{H} 10$ & 0.89350 & 0.16560 & 0.43560 & $0.1570 *$ \\
\hline H11 & 0.76210 & 0.06110 & 0.45070 & $0.1060 *$ \\
\hline H13A & 0.62230 & 0.01380 & 0.47860 & $0.0780 *$ \\
\hline H13B & 0.55450 & 0.05590 & 0.54060 & $0.0780 *$ \\
\hline $\mathrm{H} 14 \mathrm{~A}$ & 0.69250 & -0.17810 & 0.57610 & $0.0630 *$ \\
\hline H14B & 0.62810 & -0.13070 & 0.64120 & $0.0630 *$ \\
\hline H16 & 0.65840 & -0.54830 & 0.37290 & $0.0660 *$ \\
\hline $\mathrm{H} 18$ & 0.82840 & -0.25080 & 0.43000 & $0.0680 *$ \\
\hline H19 & 0.94440 & -0.20000 & 0.35810 & $0.0750 *$ \\
\hline $\mathrm{H} 21$ & 0.85520 & -0.53820 & 0.18000 & $0.0840 *$ \\
\hline $\mathrm{H} 22$ & 0.73950 & -0.59110 & 0.25280 & $0.0780 *$ \\
\hline
\end{tabular}

Atomic displacement parameters $\left(\AA^{2}\right)$

\begin{tabular}{lllllll}
\hline & $U^{11}$ & $U^{22}$ & $U^{33}$ & $U^{12}$ & $U^{13}$ & $U^{23}$ \\
\hline C11 & $0.0802(4)$ & $0.0860(4)$ & $0.1213(6)$ & $0.0009(3)$ & $0.0377(4)$ & $-0.0096(4)$ \\
O1 & $0.0627(8)$ & $0.0490(7)$ & $0.0762(9)$ & $-0.0175(6)$ & $0.0092(7)$ & $-0.0065(6)$ \\
N1 & $0.0572(9)$ & $0.0354(7)$ & $0.0690(10)$ & $-0.0128(6)$ & $-0.0100(7)$ & $-0.0014(7)$ \\
N2 & $0.0576(9)$ & $0.0383(7)$ & $0.0638(10)$ & $-0.0184(7)$ & $0.0009(7)$ & $-0.0023(7)$
\end{tabular}




\begin{tabular}{lllllll} 
& & & & & \\
$\mathrm{N} 3$ & $0.0525(8)$ & $0.0393(7)$ & $0.0605(9)$ & $-0.0101(6)$ & $-0.0020(7)$ & $0.0047(7)$ \\
C1 & $0.0650(12)$ & $0.0325(8)$ & $0.0620(11)$ & $-0.0028(8)$ & $-0.0089(9)$ & $0.0074(8)$ \\
C2 & $0.0819(16)$ & $0.0551(12)$ & $0.1050(19)$ & $0.0077(11)$ & $0.0151(14)$ & $0.0141(13)$ \\
C3 & $0.157(3)$ & $0.0816(19)$ & $0.101(2)$ & $0.041(2)$ & $0.055(2)$ & $0.0206(17)$ \\
C4 & $0.221(4)$ & $0.0656(17)$ & $0.074(2)$ & $0.013(2)$ & $0.010(2)$ & $-0.0023(15)$ \\
C5 & $0.160(3)$ & $0.0472(12)$ & $0.0692(16)$ & $-0.0157(15)$ & $-0.0327(17)$ & $0.0001(11)$ \\
C6 & $0.0858(14)$ & $0.0358(9)$ & $0.0567(11)$ & $-0.0128(9)$ & $-0.0216(10)$ & $0.0100(8)$ \\
C7 & $0.0677(12)$ & $0.0478(10)$ & $0.0745(14)$ & $-0.0214(9)$ & $-0.0224(11)$ & $0.0250(10)$ \\
C8 & $0.0827(18)$ & $0.093(2)$ & $0.133(3)$ & $-0.0414(16)$ & $-0.0290(17)$ & $0.0530(19)$ \\
C9 & $0.0716(19)$ & $0.149(3)$ & $0.191(4)$ & $-0.016(2)$ & $0.029(2)$ & $0.094(3)$ \\
C10 & $0.115(3)$ & $0.137(3)$ & $0.147(3)$ & $0.019(2)$ & $0.055(2)$ & $0.062(3)$ \\
C11 & $0.101(2)$ & $0.0791(16)$ & $0.0881(18)$ & $0.0100(15)$ & $0.0215(15)$ & $0.0222(14)$ \\
C12 & $0.0640(12)$ & $0.0444(9)$ & $0.0637(12)$ & $-0.0037(9)$ & $-0.0031(9)$ & $0.0148(9)$ \\
C13 & $0.0723(13)$ & $0.0375(9)$ & $0.0786(13)$ & $-0.0143(9)$ & $-0.0275(10)$ & $0.0013(9)$ \\
C14 & $0.0569(10)$ & $0.0389(8)$ & $0.0584(11)$ & $-0.0108(8)$ & $-0.0099(8)$ & $-0.0005(8)$ \\
C15 & $0.0580(11)$ & $0.0334(8)$ & $0.0575(11)$ & $-0.0100(8)$ & $-0.0050(8)$ & $0.0058(8)$ \\
C16 & $0.0560(11)$ & $0.0377(9)$ & $0.0682(12)$ & $-0.0101(8)$ & $-0.0073(9)$ & $-0.0006(8)$ \\
C17 & $0.0524(10)$ & $0.0370(8)$ & $0.0609(11)$ & $-0.0019(7)$ & $-0.0073(8)$ & $-0.0011(8)$ \\
C18 & $0.0584(11)$ & $0.0493(10)$ & $0.0611(11)$ & $-0.0056(9)$ & $-0.0033(9)$ & $-0.0079(9)$ \\
C19 & $0.0538(11)$ & $0.0509(10)$ & $0.0811(14)$ & $-0.0078(9)$ & $-0.0043(10)$ & $-0.0045(10)$ \\
C20 & $0.0579(11)$ & $0.0515(10)$ & $0.0783(14)$ & $0.0090(9)$ & $0.0054(10)$ & $-0.0043(10)$ \\
C21 & $0.0703(13)$ & $0.0644(13)$ & $0.0750(14)$ & $0.0045(11)$ & $0.0019(11)$ & $-0.0212(11)$ \\
C22 & $0.0606(12)$ & $0.0534(11)$ & $0.0781(14)$ & $-0.0052(9)$ & $-0.0060(10)$ & $-0.0178(10)$ \\
& & & & & & \\
\hline
\end{tabular}

Geometric parameters $\left(A,{ }^{o}\right)$

\begin{tabular}{llll}
\hline $\mathrm{C} 11-\mathrm{C} 20$ & $1.733(2)$ & $\mathrm{C} 16-\mathrm{C} 17$ & $1.453(3)$ \\
$\mathrm{O} 1-\mathrm{C} 15$ & $1.229(2)$ & $\mathrm{C} 17-\mathrm{C} 22$ & $1.384(3)$ \\
$\mathrm{N} 1-\mathrm{C} 1$ & $1.384(2)$ & $\mathrm{C} 17-\mathrm{C} 18$ & $1.394(2)$ \\
$\mathrm{N} 1-\mathrm{C} 12$ & $1.373(3)$ & $\mathrm{C} 18-\mathrm{C} 19$ & $1.374(3)$ \\
$\mathrm{N} 1-\mathrm{C} 13$ & $1.446(2)$ & $\mathrm{C} 19-\mathrm{C} 20$ & $1.370(3)$ \\
$\mathrm{N} 2-\mathrm{N} 3$ & $1.373(2)$ & $\mathrm{C} 20-\mathrm{C} 21$ & $1.374(3)$ \\
$\mathrm{N} 2-\mathrm{C} 15$ & $1.345(2)$ & $\mathrm{C} 21-\mathrm{C} 22$ & $1.380(3)$ \\
$\mathrm{N} 3-\mathrm{C} 16$ & $1.274(2)$ & $\mathrm{C} 2-\mathrm{H} 2$ & 0.9300 \\
$\mathrm{C} 1-\mathrm{C} 2$ & $1.376(3)$ & $\mathrm{C} 3-\mathrm{H} 3$ & 0.9300 \\
$\mathrm{C} 1-\mathrm{C} 6$ & $1.408(3)$ & $\mathrm{C} 4-\mathrm{H} 4$ & 0.9300 \\
$\mathrm{C} 2-\mathrm{C} 3$ & $1.404(4)$ & $\mathrm{C} 5-\mathrm{H} 5$ & 0.9300 \\
$\mathrm{~N} 2-\mathrm{H} 2 \mathrm{~N}$ & 0.8100 & $\mathrm{C} 8-\mathrm{H} 8$ & 0.9300 \\
$\mathrm{C} 3-\mathrm{C} 4$ & $1.385(5)$ & $\mathrm{C} 9-\mathrm{H} 9$ & 0.9300 \\
$\mathrm{C} 4-\mathrm{C} 5$ & $1.336(5)$ & $\mathrm{C} 10-\mathrm{H} 10$ & 0.9300 \\
$\mathrm{C} 5-\mathrm{C} 6$ & $1.389(3)$ & $\mathrm{C} 11-\mathrm{H} 11$ & 0.9300 \\
$\mathrm{C} 6-\mathrm{C} 7$ & $1.427(3)$ & $\mathrm{C} 13-\mathrm{H} 13 \mathrm{~A}$ & 0.9700 \\
$\mathrm{C} 7-\mathrm{C} 12$ & $1.405(3)$ & $\mathrm{C} 13-\mathrm{H} 13 \mathrm{~B}$ & 0.9700 \\
$\mathrm{C} 7-\mathrm{C} 8$ & $1.406(4)$ & $\mathrm{C} 14-\mathrm{H} 14 \mathrm{~A}$ & 0.9700 \\
$\mathrm{C} 8-\mathrm{C} 9$ & $1.377(5)$ & $\mathrm{C} 14-\mathrm{H} 14 \mathrm{~B}$ & 0.9300 \\
$\mathrm{C} 9-\mathrm{C} 10$ & $1.377(6)$ & $\mathrm{C} 16-\mathrm{H} 16$ & 0.9300 \\
$\mathrm{C} 10-\mathrm{C} 11$ & $1.342(6)$ & $\mathrm{C} 18-\mathrm{H} 18$ & 0.9300 \\
$\mathrm{C} 11-\mathrm{C} 12$ & $1.380(3)$ & $\mathrm{C} 19-\mathrm{H} 19$ &
\end{tabular}




\begin{tabular}{|c|c|c|c|}
\hline $\mathrm{C} 13-\mathrm{C} 14$ & $1.521(2)$ & $\mathrm{C} 21-\mathrm{H} 21$ & 0.9300 \\
\hline $\mathrm{C} 14-\mathrm{C} 15$ & $1.504(2)$ & $\mathrm{C} 22-\mathrm{H} 22$ & 0.9300 \\
\hline $\mathrm{C} 1-\mathrm{N} 1-\mathrm{C} 12$ & $109.24(15)$ & $\mathrm{C} 19-\mathrm{C} 20-\mathrm{C} 21$ & $121.2(2)$ \\
\hline $\mathrm{C} 1-\mathrm{N} 1-\mathrm{C} 13$ & $124.70(16)$ & $\mathrm{C} 20-\mathrm{C} 21-\mathrm{C} 22$ & $118.7(2)$ \\
\hline $\mathrm{C} 12-\mathrm{N} 1-\mathrm{C} 13$ & $125.17(16)$ & $\mathrm{C} 17-\mathrm{C} 22-\mathrm{C} 21$ & $121.73(19)$ \\
\hline $\mathrm{N} 3-\mathrm{N} 2-\mathrm{C} 15$ & $121.05(14)$ & $\mathrm{C} 1-\mathrm{C} 2-\mathrm{H} 2$ & 122.00 \\
\hline $\mathrm{N} 2-\mathrm{N} 3-\mathrm{C} 16$ & $116.50(14)$ & $\mathrm{C} 3-\mathrm{C} 2-\mathrm{H} 2$ & 122.00 \\
\hline $\mathrm{N} 1-\mathrm{C} 1-\mathrm{C} 2$ & $129.57(18)$ & $\mathrm{C} 2-\mathrm{C} 3-\mathrm{H} 3$ & 119.00 \\
\hline $\mathrm{N} 1-\mathrm{C} 1-\mathrm{C} 6$ & $108.41(16)$ & $\mathrm{C} 4-\mathrm{C} 3-\mathrm{H} 3$ & 119.00 \\
\hline $\mathrm{C} 2-\mathrm{C} 1-\mathrm{C} 6$ & $121.98(18)$ & $\mathrm{C} 3-\mathrm{C} 4-\mathrm{H} 4$ & 119.00 \\
\hline $\mathrm{C} 1-\mathrm{C} 2-\mathrm{C} 3$ & $116.4(2)$ & $\mathrm{C} 5-\mathrm{C} 4-\mathrm{H} 4$ & 119.00 \\
\hline $\mathrm{N} 3-\mathrm{N} 2-\mathrm{H} 2 \mathrm{~N}$ & 120.00 & $\mathrm{C} 4-\mathrm{C} 5-\mathrm{H} 5$ & 120.00 \\
\hline $\mathrm{C} 15-\mathrm{N} 2-\mathrm{H} 2 \mathrm{~N}$ & 119.00 & $\mathrm{C} 6-\mathrm{C} 5-\mathrm{H} 5$ & 120.00 \\
\hline $\mathrm{C} 2-\mathrm{C} 3-\mathrm{C} 4$ & $121.4(3)$ & $\mathrm{C} 7-\mathrm{C} 8-\mathrm{H} 8$ & 121.00 \\
\hline $\mathrm{C} 3-\mathrm{C} 4-\mathrm{C} 5$ & $121.4(3)$ & $\mathrm{C} 9-\mathrm{C} 8-\mathrm{H} 8$ & 121.00 \\
\hline $\mathrm{C} 4-\mathrm{C} 5-\mathrm{C} 6$ & $119.7(3)$ & $\mathrm{C} 8-\mathrm{C} 9-\mathrm{H} 9$ & 119.00 \\
\hline $\mathrm{C} 5-\mathrm{C} 6-\mathrm{C} 7$ & $134.3(2)$ & $\mathrm{C} 10-\mathrm{C} 9-\mathrm{H} 9$ & 119.00 \\
\hline $\mathrm{C} 1-\mathrm{C} 6-\mathrm{C} 7$ & $106.58(16)$ & $\mathrm{C} 9-\mathrm{C} 10-\mathrm{H} 10$ & 119.00 \\
\hline $\mathrm{C} 1-\mathrm{C} 6-\mathrm{C} 5$ & $119.1(2)$ & $\mathrm{C} 11-\mathrm{C} 10-\mathrm{H} 10$ & 119.00 \\
\hline $\mathrm{C} 6-\mathrm{C} 7-\mathrm{C} 8$ & $135.1(2)$ & $\mathrm{C} 10-\mathrm{C} 11-\mathrm{H} 11$ & 121.00 \\
\hline $\mathrm{C} 6-\mathrm{C} 7-\mathrm{C} 12$ & $107.36(18)$ & $\mathrm{C} 12-\mathrm{C} 11-\mathrm{H} 11$ & 121.00 \\
\hline $\mathrm{C} 8-\mathrm{C} 7-\mathrm{C} 12$ & $117.5(2)$ & $\mathrm{N} 1-\mathrm{C} 13-\mathrm{H} 13 \mathrm{~A}$ & 109.00 \\
\hline $\mathrm{C} 7-\mathrm{C} 8-\mathrm{C} 9$ & $118.3(3)$ & $\mathrm{N} 1-\mathrm{C} 13-\mathrm{H} 13 \mathrm{~B}$ & 109.00 \\
\hline $\mathrm{C} 8-\mathrm{C} 9-\mathrm{C} 10$ & $121.8(3)$ & $\mathrm{C} 14-\mathrm{C} 13-\mathrm{H} 13 \mathrm{~A}$ & 109.00 \\
\hline $\mathrm{C} 9-\mathrm{C} 10-\mathrm{C} 11$ & $121.6(4)$ & $\mathrm{C} 14-\mathrm{C} 13-\mathrm{H} 13 \mathrm{~B}$ & 109.00 \\
\hline $\mathrm{C} 10-\mathrm{C} 11-\mathrm{C} 12$ & $117.9(3)$ & $\mathrm{H} 13 \mathrm{~A}-\mathrm{C} 13-\mathrm{H} 13 \mathrm{~B}$ & 108.00 \\
\hline $\mathrm{N} 1-\mathrm{C} 12-\mathrm{C} 7$ & $108.37(17)$ & $\mathrm{C} 13-\mathrm{C} 14-\mathrm{H} 14 \mathrm{~A}$ & 110.00 \\
\hline $\mathrm{N} 1-\mathrm{C} 12-\mathrm{C} 11$ & $128.76(19)$ & $\mathrm{C} 13-\mathrm{C} 14-\mathrm{H} 14 \mathrm{~B}$ & 110.00 \\
\hline $\mathrm{C} 7-\mathrm{C} 12-\mathrm{C} 11$ & $122.9(2)$ & $\mathrm{C} 15-\mathrm{C} 14-\mathrm{H} 14 \mathrm{~A}$ & 110.00 \\
\hline $\mathrm{N} 1-\mathrm{C} 13-\mathrm{C} 14$ & $112.84(16)$ & C15-C14-H14B & 110.00 \\
\hline $\mathrm{C} 13-\mathrm{C} 14-\mathrm{C} 15$ & $110.00(15)$ & $\mathrm{H} 14 \mathrm{~A}-\mathrm{C} 14-\mathrm{H} 14 \mathrm{~B}$ & 108.00 \\
\hline $\mathrm{N} 2-\mathrm{C} 15-\mathrm{C} 14$ & $118.09(16)$ & $\mathrm{N} 3-\mathrm{C} 16-\mathrm{H} 16$ & 120.00 \\
\hline $\mathrm{O} 1-\mathrm{C} 15-\mathrm{C} 14$ & $121.57(16)$ & $\mathrm{C} 17-\mathrm{C} 16-\mathrm{H} 16$ & 120.00 \\
\hline $\mathrm{O} 1-\mathrm{C} 15-\mathrm{N} 2$ & $120.28(16)$ & $\mathrm{C} 17-\mathrm{C} 18-\mathrm{H} 18$ & 120.00 \\
\hline $\mathrm{N} 3-\mathrm{C} 16-\mathrm{C} 17$ & $120.91(15)$ & $\mathrm{C} 19-\mathrm{C} 18-\mathrm{H} 18$ & 120.00 \\
\hline $\mathrm{C} 18-\mathrm{C} 17-\mathrm{C} 22$ & $117.87(17)$ & $\mathrm{C} 18-\mathrm{C} 19-\mathrm{H} 19$ & 120.00 \\
\hline $\mathrm{C} 16-\mathrm{C} 17-\mathrm{C} 18$ & $122.40(16)$ & $\mathrm{C} 20-\mathrm{C} 19-\mathrm{H} 19$ & 120.00 \\
\hline $\mathrm{C} 16-\mathrm{C} 17-\mathrm{C} 22$ & $119.72(16)$ & $\mathrm{C} 20-\mathrm{C} 21-\mathrm{H} 21$ & 121.00 \\
\hline $\mathrm{C} 17-\mathrm{C} 18-\mathrm{C} 19$ & $120.82(18)$ & $\mathrm{C} 22-\mathrm{C} 21-\mathrm{H} 21$ & 121.00 \\
\hline $\mathrm{C} 18-\mathrm{C} 19-\mathrm{C} 20$ & $119.68(19)$ & $\mathrm{C} 17-\mathrm{C} 22-\mathrm{H} 22$ & 119.00 \\
\hline $\mathrm{C} 11-\mathrm{C} 20-\mathrm{C} 21$ & $119.48(17)$ & $\mathrm{C} 21-\mathrm{C} 22-\mathrm{H} 22$ & 119.00 \\
\hline $\mathrm{C} 11-\mathrm{C} 20-\mathrm{C} 19$ & $119.30(16)$ & & \\
\hline $\mathrm{C} 1-\mathrm{N} 1-\mathrm{C} 12-\mathrm{C} 7$ & $-2.1(2)$ & $\mathrm{C} 5-\mathrm{C} 6-\mathrm{C} 7-\mathrm{C} 8$ & $-0.5(4)$ \\
\hline $\mathrm{C} 12-\mathrm{N} 1-\mathrm{C} 1-\mathrm{C} 2$ & $-175.4(2)$ & $\mathrm{C} 6-\mathrm{C} 7-\mathrm{C} 12-\mathrm{N} 1$ & $1.2(2)$ \\
\hline $\mathrm{C} 13-\mathrm{N} 1-\mathrm{C} 1-\mathrm{C} 2$ & $-5.7(3)$ & $\mathrm{C} 6-\mathrm{C} 7-\mathrm{C} 12-\mathrm{C} 11$ & $-178.4(2)$ \\
\hline $\mathrm{C} 12-\mathrm{N} 1-\mathrm{C} 1-\mathrm{C} 6$ & $2.3(2)$ & $\mathrm{C} 6-\mathrm{C} 7-\mathrm{C} 8-\mathrm{C} 9$ & $177.5(3)$ \\
\hline
\end{tabular}




$\begin{array}{llll}\mathrm{C} 13-\mathrm{N} 1-\mathrm{C} 1-\mathrm{C} 6 & 171.93(16) & \mathrm{C} 12-\mathrm{C} 7-\mathrm{C} 8-\mathrm{C} 9 & -0.2(4) \\ \mathrm{C} 12-\mathrm{N} 1-\mathrm{C} 13-\mathrm{C} 14 & 84.8(2) & \mathrm{C} 8-\mathrm{C} 7-\mathrm{C} 12-\mathrm{N} 1 & 179.5(2) \\ \mathrm{C} 1-\mathrm{N} 1-\mathrm{C} 13-\mathrm{C} 14 & -83.2(2) & \mathrm{C} 8-\mathrm{C} 7-\mathrm{C} 12-\mathrm{C} 11 & -0.1(3) \\ \mathrm{C} 13-\mathrm{N} 1-\mathrm{C} 12-\mathrm{C} 7 & -171.72(17) & \mathrm{C} 7-\mathrm{C} 8-\mathrm{C} 9-\mathrm{C} 10 & -0.1(6) \\ \mathrm{C} 1-\mathrm{N} 1-\mathrm{C} 12-\mathrm{C} 11 & 177.4(2) & \mathrm{C} 8-\mathrm{C} 9-\mathrm{C} 10-\mathrm{C} 11 & 0.8(7) \\ \mathrm{C} 13-\mathrm{N} 1-\mathrm{C} 12-\mathrm{C} 11 & 7.8(3) & \mathrm{C} 9-\mathrm{C} 10-\mathrm{C} 11-\mathrm{C} 12 & -1.1(6) \\ \mathrm{C} 15-\mathrm{N} 2-\mathrm{N} 3-\mathrm{C} 16 & 178.89(16) & \mathrm{C} 10-\mathrm{C} 11-\mathrm{C} 12-\mathrm{C} 7 & 0.7(4) \\ \mathrm{N} 3-\mathrm{N} 2-\mathrm{C} 15-\mathrm{C} 14 & -0.5(2) & \mathrm{C} 10-\mathrm{C} 11-\mathrm{C} 12-\mathrm{N} 1 & -178.7(3) \\ \mathrm{N} 3-\mathrm{N} 2-\mathrm{C} 15-\mathrm{O} 1 & 176.58(16) & \mathrm{N} 1-\mathrm{C} 13-\mathrm{C} 14-\mathrm{C} 15 & 177.03(16) \\ \mathrm{N} 2-\mathrm{N} 3-\mathrm{C} 16-\mathrm{C} 17 & 178.32(15) & \mathrm{C} 13-\mathrm{C} 14-\mathrm{C} 15-\mathrm{O} 1 & -88.0(2) \\ \mathrm{N} 1-\mathrm{C} 1-\mathrm{C} 6-\mathrm{C} 5 & -179.18(18) & \mathrm{C} 13-\mathrm{C} 14-\mathrm{C} 15-\mathrm{N} 2 & 89.05(19) \\ \mathrm{N} 1-\mathrm{C} 1-\mathrm{C} 6-\mathrm{C} 7 & -1.5(2) & \mathrm{N} 3-\mathrm{C} 16-\mathrm{C} 17-\mathrm{C} 18 & 11.7(3) \\ \mathrm{C} 6-\mathrm{C} 1-\mathrm{C} 2-\mathrm{C} 3 & -0.1(3) & \mathrm{N} 3-\mathrm{C} 16-\mathrm{C} 17-\mathrm{C} 22 & -167.34(18) \\ \mathrm{N} 1-\mathrm{C} 1-\mathrm{C} 2-\mathrm{C} 3 & 177.3(2) & \mathrm{C} 16-\mathrm{C} 17-\mathrm{C} 18-\mathrm{C} 19 & -177.55(17) \\ \mathrm{C} 2-\mathrm{C} 1-\mathrm{C} 6-\mathrm{C} 7 & 176.36(19) & \mathrm{C} 22-\mathrm{C} 17-\mathrm{C} 18-\mathrm{C} 19 & 1.5(3) \\ \mathrm{C} 2-\mathrm{C} 1-\mathrm{C} 6-\mathrm{C} 5 & -1.3(3) & \mathrm{C} 16-\mathrm{C} 17-\mathrm{C} 22-\mathrm{C} 21 & 177.23(19) \\ \mathrm{C} 1-\mathrm{C} 2-\mathrm{C} 3-\mathrm{C} 4 & 1.5(4) & \mathrm{C} 18-\mathrm{C} 17-\mathrm{C} 22-\mathrm{C} 21 & -1.9(3) \\ \mathrm{C} 2-\mathrm{C} 3-\mathrm{C} 4-\mathrm{C} 5 & -1.5(5) & \mathrm{C} 17-\mathrm{C} 18-\mathrm{C} 19-\mathrm{C} 20 & 0.0(3) \\ \mathrm{C} 3-\mathrm{C} 4-\mathrm{C} 5-\mathrm{C} 6 & 0.0(5) & \mathrm{C} 18-\mathrm{C} 19-\mathrm{C} 20-\mathrm{C} 11 & 178.96(16) \\ \mathrm{C} 4-\mathrm{C} 5-\mathrm{C} 6-\mathrm{C} 7 & -175.5(3) & \mathrm{C} 18-\mathrm{C} 19-\mathrm{C} 20-\mathrm{C} 21 & -1.3(3) \\ \mathrm{C} 4-\mathrm{C} 5-\mathrm{C} 6-\mathrm{C} 1 & 1.4(4) & \mathrm{C} 11-\mathrm{C} 20-\mathrm{C} 21-\mathrm{C} 22 & -179.29(17) \\ \mathrm{C} 5-\mathrm{C} 6-\mathrm{C} 7-\mathrm{C} 12 & 177.4(2) & \mathrm{C} 19-\mathrm{C} 20-\mathrm{C} 21-\mathrm{C} 22 & 1.0(3) \\ \mathrm{C} 1-\mathrm{C} 6-\mathrm{C} 7-\mathrm{C} 12 & 0.2(2) & \mathrm{C} 20-\mathrm{C} 21-\mathrm{C} 22-\mathrm{C} 17 & 0.7(3) \\ \mathrm{C} 1-\mathrm{C} 6-\mathrm{C} 7-\mathrm{C} 8 & -177.6(3) & & \end{array}$

Hydrogen-bond geometry $\left(A,{ }^{\circ}\right)$

$\mathrm{Cg} 2, \mathrm{Cg} 3$ and $\mathrm{Cg} 4$ are the centroids of the two benzene rings (C1-C6 and $\mathrm{C} 7-\mathrm{C} 12)$ of the carbazole ring system and the chlorophenyl ring (C17- $\mathrm{C} 22)$, respectively.

\begin{tabular}{lllll}
\hline$D-\mathrm{H} \cdots A$ & $D-\mathrm{H}$ & $\mathrm{H} \cdots A$ & $D \cdots A$ & $D-\mathrm{H} \cdots A$ \\
\hline $\mathrm{N} 2-\mathrm{H} 2 N \cdots \mathrm{O} 1^{\mathrm{i}}$ & 0.81 & 2.08 & $2.8952(19)$ & 175 \\
$\mathrm{C} 14-\mathrm{H} 14 A \cdots \mathrm{N} 3$ & 0.97 & 2.42 & $2.765(2)$ & 100 \\
$\mathrm{C} 5-\mathrm{H} 5 \cdots C g 4^{\mathrm{ii}}$ & 0.93 & 2.81 & $3.696(3)$ & 160 \\
$\mathrm{C} 21-\mathrm{H} 21 \cdots C g 3^{\mathrm{iii}}$ & 0.93 & 2.97 & $3.858(3)$ & 160 \\
$\mathrm{C} 22-\mathrm{H} 22 \cdots C g 2^{\mathrm{iii}}$ & 0.93 & 2.79 & $3.699(2)$ & 166 \\
\hline
\end{tabular}

Symmetry codes: (i) $-x+1,-y-1,-z+1$; (ii) $x,-y+1 / 2, z+1 / 2$; (iii) $x,-y-1 / 2, z-1 / 2$. 